\title{
An attempt to estimate out-of-plane lung nodule elongation in tomosynthesis images
}

\author{
Artur Chodorowski ${ }^{1,2}$, Jonathan Arvidsson ${ }^{3}$, Christina Söderman ${ }^{4}$, Angelica Svalkvist ${ }^{3}$, \\ Åse A. Johnsson ${ }^{5,6}$, Magnus Båth ${ }^{3,4}$ \\ ${ }^{1}$ Signals and Systems, Chalmers University of Technology, Gothenburg, Sweden \\ ${ }^{2}$ MedTech West, Sahlgrenska University Hospital, Gothenburg, Sweden \\ ${ }^{3}$ Department of Medical Physics and Biomedical Engineering, Sahlgrenska University Hospital, \\ Gothenburg, Sweden \\ ${ }^{4}$ Department of Radiation Physics, Institute of Clinical Sciences, Sahlgrenska Academy, University \\ of Gothenburg, Gothenburg, Sweden \\ ${ }^{5}$ Department of Radiology, Institute of Clinical Sciences, Sahlgrenska Academy, University of \\ Gothenburg, Gothenburg, Sweden \\ ${ }^{6}$ Department of Radiology, Sahlgrenska University Hospital, Gothenburg, Sweden
}

\begin{abstract}
In chest tomosynthesis (TS) the most commonly used reconstruction methods are based on Filtered Back Projection (FBP) algorithms. Due to the limited angular range of $\mathrm{x}$-ray projections, FBP reconstructed data is typically associated with a low spatial resolution in the out-of-plane dimension. Lung nodule measures that depend on depth information such as 3D shape and volume are therefore difficult to estimate.

In this paper the relation between features from FBP reconstructed lung nodules and the true out-of-plane nodule elongation is investigated and a method for estimating the out-of-plane nodule elongation is proposed. In order to study these relations a number of steps that include simulation of spheroidal-shaped nodules, insertion into synthetic data volumes, construction of TS-projections and FBP-reconstruction were performed. In addition, the same procedure was used to simulate nodules and insert them into clinical chest TS projection data. The reconstructed nodule data was then investigated with respect to in-plane diameter, out-of-plane elongation, and attenuation coefficient.

It was found that the voxel value in each nodule increased linearly with nodule elongation, for nodules with a constant attenuation coefficient. Similarly, the voxel value increased linearly with in-plane diameter. These observations indicate the possibility to predict the nodule elongation from the reconstructed voxel intensity values. Such a method would represent a quantitative approach to chest tomosynthesis that may be useful in future work on volume and growth rate estimation of lung nodules.
\end{abstract}

Keywords: chest tomosynthesis, pulmonary nodules, 3D reconstruction, filtered back projection, volumetry

\section{INTRODUCTION}

Tomosynthesis (TS) is a 3D imaging technique that uses multiple radiographic images taken from an X-ray source placed at several positions [1]. The technique has similarities with Computed Tomography (CT), but among the differences is that a limited angular range of $\mathrm{x}$-ray projections is used. Furthermore, the TS-radiation dose has been shown to be substantially lower than the radiation dose in conventional chest CT [2], which makes TS an interesting alternative to CT. In chest radiology the reconstructed volumes are used for e.g. detection of pulmonary nodules and for estimation of volume and growth rate of nodules [3]. The characteristic called "volume doubling time" (the interval it takes for a

Medical Imaging 2015: Physics of Medical Imaging, edited by Christoph Hoeschen, Despina Kontos, Proc. of SPIE Vol. 9412, 94122J · (C) 2015 SPIE · CCC code: 1605-7422/15/\$18 - doi: 10.1117/12.2079901 
nodule to double in volume) is of particular interest as it is regarded as critical for cancerous nodules. The correct estimation of nodule size and shape is thus important in clinical practice [4].

In TS, contrary to CT, the correct estimation of nodule shape and volume may be difficult due to the low depth resolution resulting from the limited angular range of the projections and limitations in the reconstruction techniques $[5,6]$. It would be desirable if the reconstructed voxel values would represent a true 3D distribution of $\mathrm{x}$-ray attenuation. However, this is often not the case. In particular, in the commonly used TS reconstruction method Filtered Backprojection [7] is not obvious how to relate the reconstructed voxel values to the investigated tissue properties.

An attempt to make the FBP reconstructed images quantitative and relate them to tissue properties was performed by Shafer et al. [8], in the context of breast tomosynthesis. They studied the reconstructed voxel values (VVs) as a function of tissue glandularity for different $\mathrm{x}$-ray tube energies and different beam exposure levels, using cuboidal phantoms, and reported linear relations between VVs and the investigated parameters. There exist methods for automated 3D nodule segmentation and nodule growth rate estimation for conventional CT, see e.g. Reeves et al. [9]. However, to the authors' knowledge there exists no method that correctly performs the nodule segmentation in 3D for the FBP-based TS reconstruction. A study of lung nodules in tomosynthesis was done by Orbán et al. [10] in the context of nodule detection, but no nodule segmentation was performed. Arvidsson et al. [11] presented a method of automated nodule shape estimation, but the work was limited to in-plane segmentation and no attempt at shape estimation in 3D was made.

The purpose of the present paper was to perform an initial investigation of the possibility to predict the out-of-plane lung nodule elongation in tomosynthesis images. To achieve this we extend the study in Shafer et al. [8] and investigate the relations between the reconstructed VVs and a number of input parameters such as tissue attenuation and tissue shape. We simulate nodules of different shapes and study resulting reconstructed VVs as a function of nodule size and elongation, for different attenuation coefficients. As the reconstructed volumes are dependent on a particular 3Dreconstruction technique, we limit the experiments in this work to the conventional FBP reconstruction technique [12, 13 ] only, commonly used at clinical practice.

The found relations show that the voxel value in each nodule increased approximately linearly with nodule elongation, for nodules with a constant attenuation coefficient. Similarly, the voxel value increased approximately linearly with inplane diameter. These observations indicate the possibility to predict the nodule elongation from the reconstructed voxel intensity values. The found relations may be useful in future work on volume and growth rate estimation of lung nodules.

\section{METHODS}

In this section we briefly describe the shape of simulated nodules, the tomosynthesis system used in the study and the setup of the reconstruction experiments.

\subsection{Nodule simulation}

The simulated nodules of spheroidal shape (see Figure 1) were inserted into the raw-data TS projection images, before reconstruction of the resulting section images. The nodules were positioned at the desired location in 3D space and projected into the raw-data projection images of the TS acquisition, according to Svalkvist et al. [14, 15].
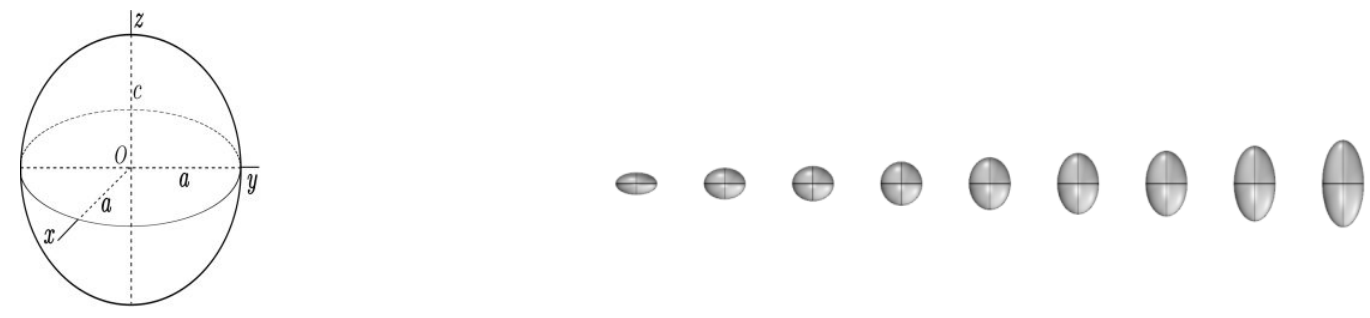

Figure 1. (left) Illustration of spheroidal shape. $\mathrm{c}$ is semi-axis length along $\mathrm{z}$-direction and a is semi-axis in $\mathrm{x}$ - or $\mathrm{y}$ - direction. The ellipsoid is oblate for $\mathrm{c} / \mathrm{a}<1$ and prolate for $\mathrm{c} / \mathrm{a}>1$. The $\mathrm{z}$-axis factor is defined as $\mathrm{c} / \mathrm{a}$ and is a measure of nodule elongation in $\mathrm{z}$ direction. (right) The simulated nodules with $\mathrm{z}$-axis factor (elongation) of $0.5,0.7,0.8,1.0,1.2,1.4,1.5,1.7$ and 2.0 (from left to right). 


\subsection{Tomosynthesis system and reconstruction}

The clinical images used in this study were acquired using the TS system GE Definium 8000 X-ray system with VolumeRAD software (GE Healthcare, Chalfont St. Giles, UK). The system collects 60 low-dose projection images within approximately 10 seconds. The detector position is fixed, whereas the X-ray tube performs a vertical movement relative to the standard orthogonal posteroanterior (PA) projection. The projection images are collected in the angular interval of $-15^{\circ}$ to $+15^{\circ}$. The focus-detector distance is $180 \mathrm{~cm}$ in the PA projection. The detector size is $2022 \times 2022$ pixels, with a pixel size of $0.2 \times 0.2 \mathrm{~mm}^{2}$. The focal spot size is $1.25 \times 1.25 \mathrm{~mm}^{2}$. Reconstruction was performed with 1 $\mathrm{mm}$ slice interval using the software VolumeRAD, which incorporates a 3D view-weighted cone-beam filtered back projection (3D VW FBP) algorithm [12, 13].

The geometry of the tomosynthesis system used in our simulation experiments is shown in Figure 2.

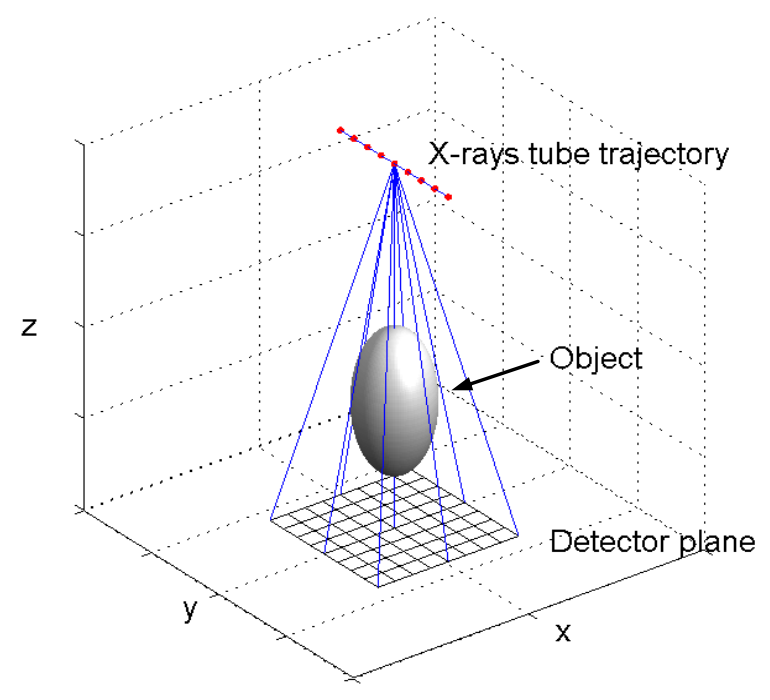

Figure 2. The geometry of the cone-beam flat-panel detector tomosynthesis system.

\subsection{Reconstruction experiments}

Two reconstruction experiments were performed, with nodules positioned in (1) noiseless background and (2) clinical/anatomical background:

Case (1) Synthetic spheroidal nodules with diameter of 4, 5, 6, 7, $8 \mathrm{~mm}$ and varying z-axis factor, inserted in a noiseless background.

Case (2) Synthetic spheroidal nodules with diameter $7 \mathrm{~mm}$ and varying $\mathrm{z}$-axis factor, inserted in a clinical data with anatomically homogeneous background (see Figure 3). The choice of diameter value of $7 \mathrm{~mm}$ was motivated by the fact that such a nodule has a volume in the middle of the range of $100-300 \mathrm{~mm}^{3}$, which is regarded as the clinically most relevant range for follow up [16].

The elongation of the nodules (z-axis factor) in both cases varied with $0.5,0.7,0.8,1.0,1.2,1.4,1.5,1.7$ and 2.0. The nodule attenuation coefficients $0.017,0.023$ and $0.027 \mathrm{~mm}^{-1}$ were used. The computer simulations were done using Matlab [17] and Rhinoceros [18] software. 


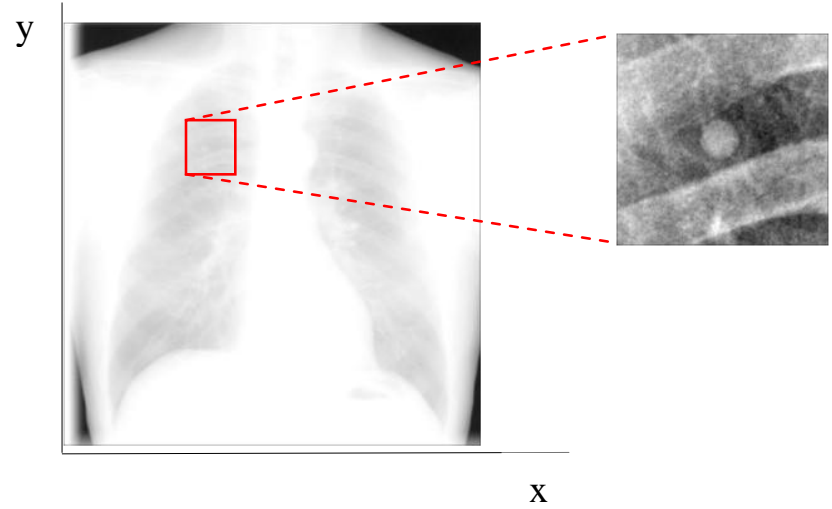

Figure 3. An example of a tomosynthesis projection image (detector primary angle $0.35^{\circ}$ ) of clinical data with an inserted synthetic nodule. Nodule diameter $7 \mathrm{~mm}$, elongation 1.4, attenuation coefficient $0.017 \mathrm{~mm}^{-1}$. The zoomed image on the right represents the intensity stretched ROI (the red box).

\section{RESULTS AND DISCUSSION}

Figure 4-8 show sections of reconstructed nodules in different planes and the found relations between the input parameters and the reconstructed voxel values, for the abovementioned cases (1) and (2).

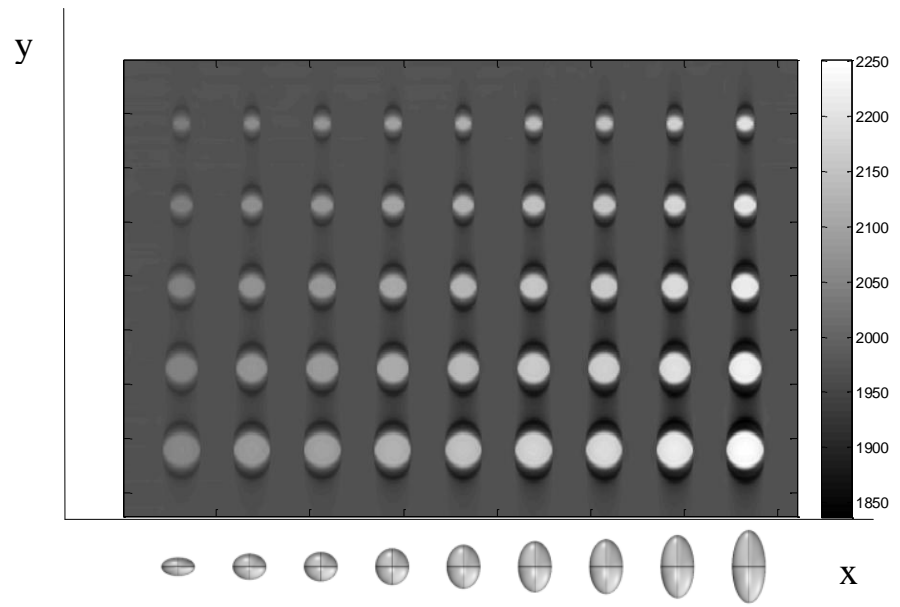

Figure 4. The VVs of the central X-Y plane of the reconstructed nodules, noiseless background (case 1). The nodules are arranged in ascending order according to their diameter and elongation. The bottommost row illustrates how the ellipsoidal z-axis factor varies in ascending order with values $0.5,0.7,0.8,1.0,1.2,1.4$. 1.5, 1.7, and 2.0 (left to right). 

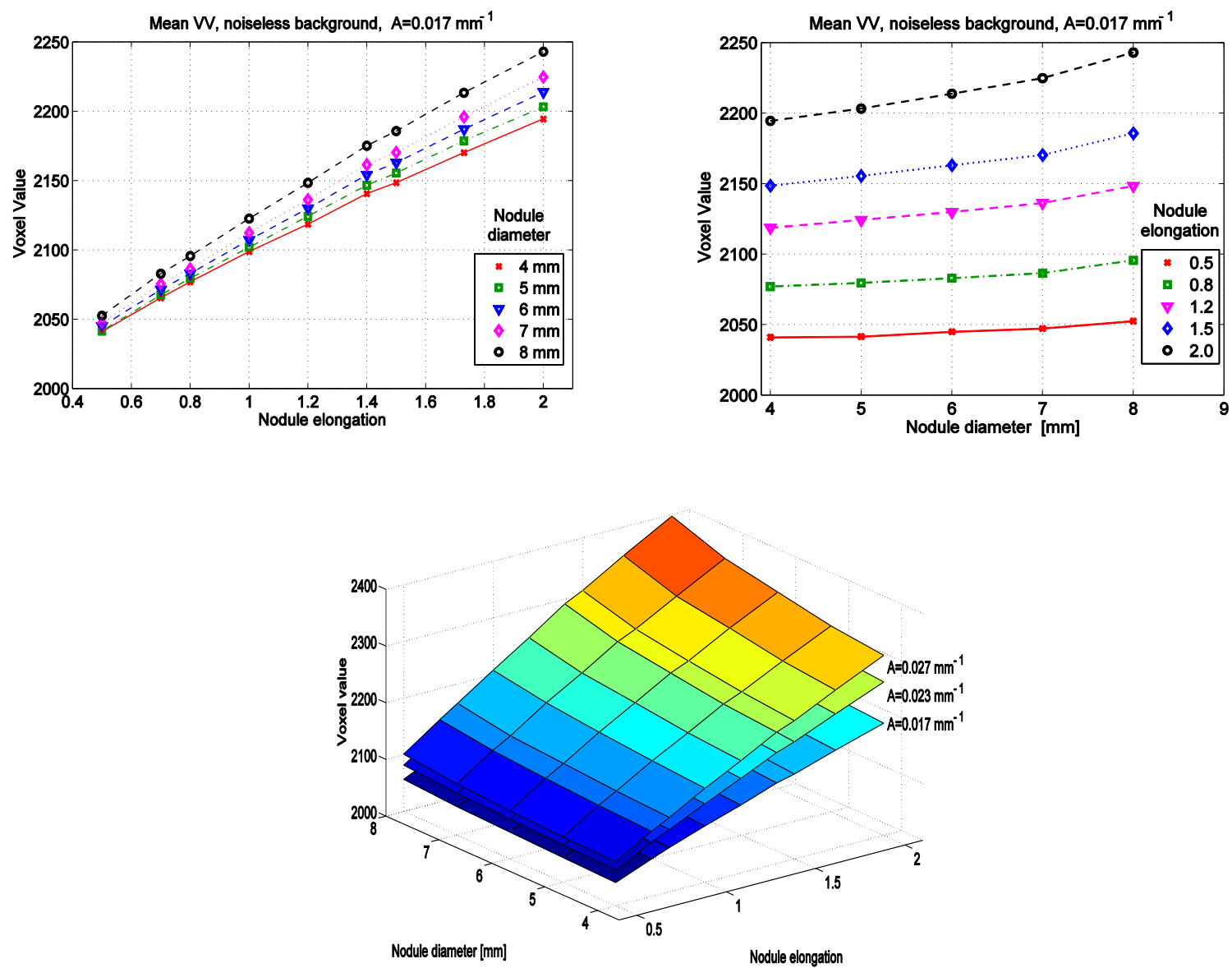

Figure 5. Case 1: noiseless background. (left) VV versus nodule elongation (right) VV versus nodule diameter. Attenuation coefficient $0.017 \mathrm{~mm}^{-1}$. (bottom) VV versus nodule in-plane diameter and z-axis elongation factor shown as regression surfaces for different nodule attenuation coefficients $\left(A=0.017,0.023,0.027 \mathrm{~mm}^{-1}\right)$. Here, the VVs represent the mean intensity value in a circular ROI in the central reconstructed slice of the nodule. The ROI was positioned in the center of the nodule and with a radius equal to half of the radius of the nodule.

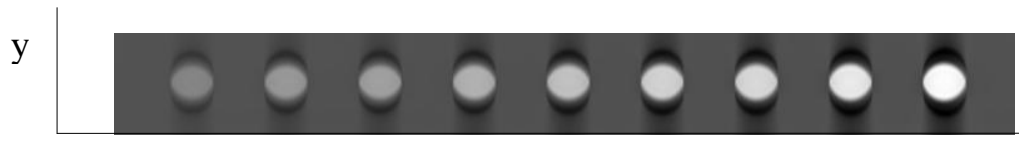

$\mathrm{X}$

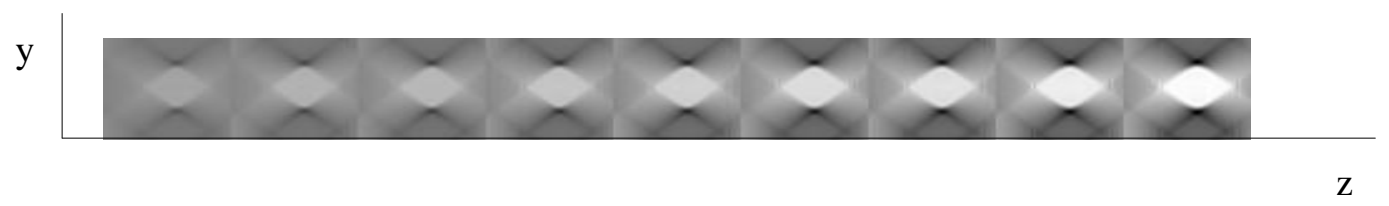

Figure 6. Case 1: noiseless background, (upper) The central XY-section of the reconstructed nodules with diameter $7 \mathrm{~mm}$ and different elongations. (lower) The corresponding YZ-section (pixel aspect ratio 2:5) of the nodules. Note that the nodule shape in z-direction (depth) is hidden within the rhombus-like structure. 

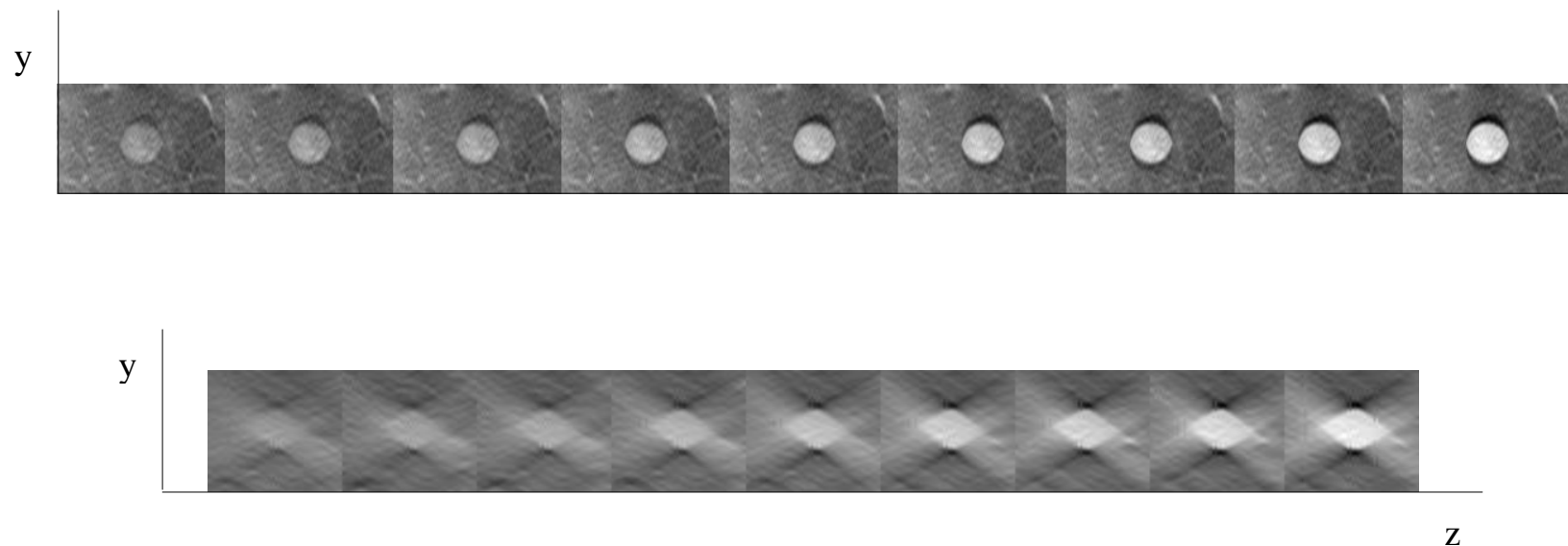

Figure 7. Case 2: clinical background. (upper) The central XY section of the reconstructed spheroidal nodules with diameter 7 mm. (lower) The corresponding YZ-section (pixel aspect ratio 2:5) of the nodules. For visualization purpose the pixel values are stretched between 0 and 1 .
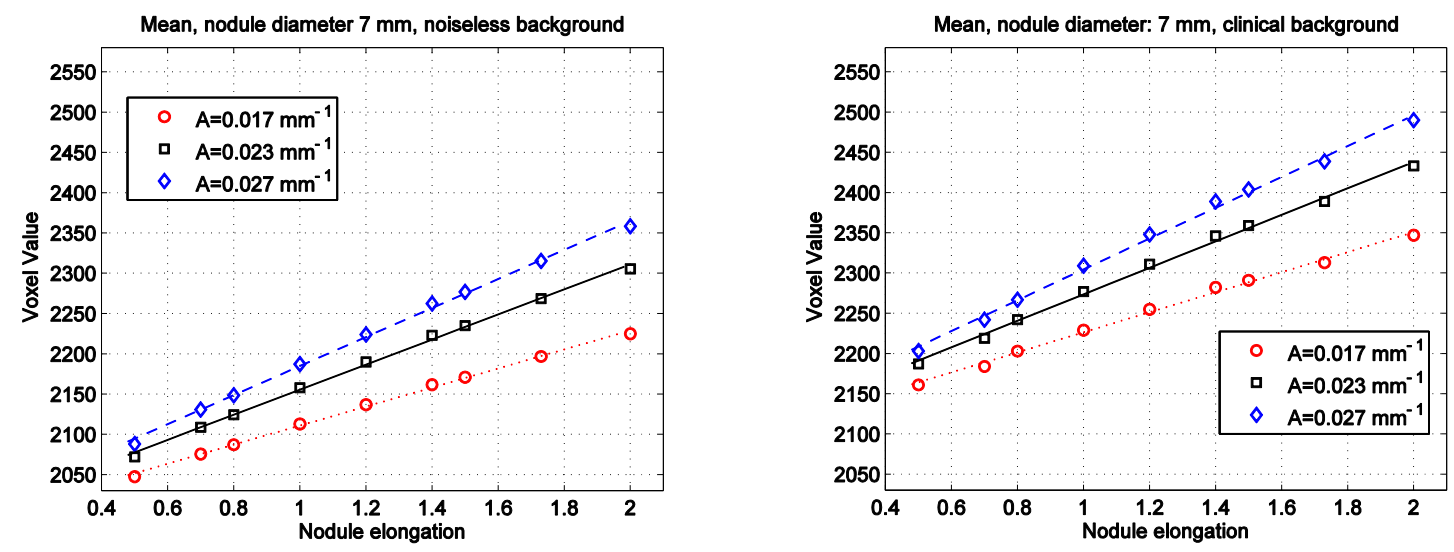

Figure 8. Least-squares linear regression curves for VV versus nodule elongation for nodule with 7 mm diameter. (left) Case 1: noiseless background. (right) Case 2: clinical/anatomical background.

Figures 4-8 demonstrate that the nodule intensity (voxel value) was dependent on nodule size in the central X-Y plane, nodule elongation along z-axis, and nodule attenuation coefficient. They also show the ambiguity between the nodule attenuation coefficient and nodule elongation. In both cases (noiseless and clinical/anatomical) the VVs increased approximately linearly with nodule diameter, nodule elongation and nodule attenuation coefficient. The sectional YZimages (Figures 6,7) show that the nodule shape in the depth direction is hidden in the high intensity rhombus-like structure and not easy to be used for inferring the nodule elongation. However, the regression relations presented in this section suggest that in some circumstances they can be used for nodule growth rate estimation. Consider the following scenario:

Time-1:

- a potential nodule is detected at some position in the patient's chest

- the position and diameter of the nodule in the central plane is estimated from the TS-reconstructed volume

- a number of nodule simulations are done to obtain regression curves and surfaces (such as in Figure 8) for this particular nodule position and the patient 
Time-2:

- the same nodule is observed at the next TS-investigation

- the intensity and nodule diameter is estimated from the reconstructed central plane of the nodule

- the regression curves are used to estimate the nodule shape, depending on different assumptions (e.g. constant attenuation factor of the nodule)

- the nodule volume is used to estimate the nodule grow rate

For example, for an attenuation equal to $0.017 \mathrm{~mm}^{-1}$, a change in $\mathrm{VV}$ by 50 units corresponds to an increase in nodule elongation by approximately 0.4 (Figure 8 ), which in turn corresponds to a change in nodule volume by a factor of 0.4 $(40 \%)$ as well, since the theoretical value for volume of a spheroidal object is equal (4/3) $\pi a^{2} c$. This calculation requires that the attenuation coefficient of the nodule is the same at the two time points and that the TS system is linear in the sense that, apart from stochastic fluctuations, the same input at two different time points results in the same output.

It should be noted that no attempt at evaluating the method of estimating nodule growth presented above was made, and the present work should be seen only as presenting a possible basis for such a method, Also, whether the simulations needed to obtain relevant regression curves and surfaces can be performed based on clinical data remains to be seen. Nevertheless, several possible difficulties may already be foreseen. The current study takes into account spheroidal nodules only. An additional simulation study may be needed to evaluate whether the current results using spheroidal nodules may be useful for volume and growth rate estimation of other nodules. Also, the simulation of nodules in nonhomogeneous anatomical backgrounds will be required for more real-case scenarios. The system transfer function and scattering were not considered in our simulations. The scattering would decrease the contrast between lesions/nodules and background and reconstructed VVs would be lower. It should also be emphasized that the presented results are valid only for the particular FBP-reconstruction method and its implementation. Another difficulty for practical use of the found regression curves may be related to the calibration problem: how to acquire two or more TS scans with the same scanner parameters. Similar regression curves could be found directly from the individual projection images where the nodule pixel values are directly related to the x-rays path length and attenuation. However, this would require selection of projection images where the nodule is not hidden by other anatomical structures. In addition, it is much more difficult to detect small nodules on the individual projection images in a noisy anatomical background.

\section{CONCLUSIONS}

It was found that the voxel values of reconstructed spheroidal nodules in a noiseless background vary depending on outof-plane elongation for nodules with constant attenuation coefficient. A similar relation was found for nodules simulated in an anatomically homogeneous background in clinical data. The results indicate the possibility of basing a method of estimating nodule elongation on reconstructed voxel intensity values. Such a method would represent a quantitative approach to chest tomosynthesis that may be useful in future work on volume and growth rate estimation of lung nodules.

\section{ACKNOWLEDGMENTS}

This work was supported by grants from the Swedish Research Council [2013/3477], the Swedish Radiation Safety Authority [2013-2982, 2014-2641], and the Swedish Federal Government under the LUA/ALF agreement [ALFGBG136281, ALFGBG-428961].

\section{REFERENCES}

1. Dobbins, III J. T., "Tomosynthesis imaging: At a translational crossroads," Med. Phys., 36(6), 1956-1967 (2009).

2. Båth, M., Svalkvist, A., von Wrangel, A., Rismyhr-Olsson, H., and Cederblad, Å., "Effective dose to patients from chest examinations with tomosynthesis," Radiat Prot Dosimetry, 139, 153-158 (2010).

3. Ko, J. P., Berman, E. J., Kaur, M. et al., "Pulmonary Nodules: Growth Rate Assessment in Patients by Using Serial CT and Three-dimensional Volumetry," Radiology, 262(2), 662-672 (2012).

4. Soardi, G. A., Perandini, S., Motton, M., and Montemezzi, S., "Assessing probability of malignancy in solid solitary pulmonary nodules with a new Bayesian calculator: improving diagnostic accuracy by means of expanded and updated features," Eur Radiol., 25(1), 155-162 (2015). 
5. Johnsson, Å. A., Fagman, E., Vikgren, J. et al., "Pulmonary nodule size evaluation with chest tomosynthesis," Radiology, 265, 273-282 (2012).

6. Johnsson, A. A., Vikgren, J., and Båth, M., "Chest tomosynthesis: technical and clinical principles," Semin. Respir. Crit. Care Med., 35, 17-26 (2014).

7. Lauritsch, G., and Härer, W. H., "A theoretical framework for filtered backprojection in tomosynthesis," Proc. SPIE 3338, 1127-1137 (1998).

8. Shafer, C. M., Samei, E., Saunders, R. S., Zerhouni, M., and Lo, J. Y., "Toward quantification of breast tomosynthesis imaging," Proc. SPIE 6913, 69134N (2008).

9. Reeves, A. P., Chan, A. B., Yankelewitz, D. F., Henschke, C., Kressler, B., and Kostis, W. J., "On Measuring the Change in Size of Pulmonary Nodules", IEEE Trans on Medical Imaging, Vol. 25, No.4, 435-450 (2006).

10. Orbán, G., and Horváth, G., "Lung Nodule Detection on Digital Tomosynthesis Images: A Preliminary Study", IEEE 14th Int Conf on Biomedical Imaging (ISBI), 141-144 (2014).

11. Arvidsson, J., Chodorowski, A., Söderman, C., Svalkvist, A., Johnsson, Å. A., and Båth, M., "Automated Estimation of In-plane Nodule Shape in Chest Tomosynthesis Images", IFMBE Proceedings of 16th NordicBaltic Conference on Biomedical Engineering, Vol. 48, (2014).

12. Li, B., Avinash, G. B., Claus, B., and Metz, S. W., "3-D Weighted Cone-beam Filtered Backprojection Reconstruction for Digital Tomosynthesis," Proc. SPIE 6510, 65104X (2007).

13. Hsieh, J., Metz, S. W., Avinash, G. B., Li, B., and Sabol, J. M., "Systems, methods and apparatus for specialized filtered back-projection reconstruction for digital tomosynthesis," US Patent US 20080107324 A1, (2008).

14. Svalkvist, A., Håkansson, M., Ullman, G., and Båth, M., "Simulation of lung nodules in chest tomosynthesis," Rad Prot Dosimetry, 193, 130-139 (2010).

15. Svalkvist, A., Johnsson, ̊. A., Vikgren, J. et al., "Evaluation of an improved method of simulating lung nodules in chest tomosynthesis," Acta Radiol., 53, 874-884 (2012).

16. Horeweg, N., van Rosmalen, J., Heuvelmans, M. A. et al., "Lung cancer probability in patients with CTdetected pulmonary nodules: a prespecified analysis of data from the NELSON trial of low-dose CT screening," Lancet Oncol., 15(12), 1332-41 (2014).

17. MATLAB Image Processing Toolbox User's Guide, The Mathworks Inc., Natick, Massachusetts, www.mathworks.com, (2014).

18. Rhinoceros 3D, Robert McNeel \& Associates, www.rhino3d.com, (2014). 